\title{
Right Hepatectomy in Post Bariatric Surgery Living Donor: Case Report
}

\author{
Manoj Gupta*, Bhushan Bhole and KR Vasudevan \\ Department of Liver Transplant and Surgical Gastroenterology, PSRI Hospital, New \\ Delhi, India \\ *Corresponding Author: Manoj Gupta, Department of Liver Transplant and \\ Surgical Gastroenterology, PSRI Hospital, New Delhi, India.
}

Received: April 08, 2021

Published: May 31, 2021

(C) All rights are reserved by Manoj Gupta., et al.

\begin{abstract}
Background: Hepatic steatosis is one of the most common causes of an increasing prevalence of non-alcoholic fatty liver disease (NAFLD) [1]. NAFLD is one of the most common causes of chronic liver disease. Weight reduction by bariatric surgery can prevent the progression of NAFLD to chronic liver disease.

Case Report: Liver donor was 50 year old female who donated for her husband. She was having past history of laparoscopic sleeve gastrectomy and her recent BMI was more than 35. On evaluation, USG abdomen was normal. MR fat estimation showed 3 - 5\% fat fraction in her right and left lobe (Picture 1). Ct volumetric analysis showed adequate remnant and graft volume. Liver biopsy analysis showed 5 - 7\% fat fraction without any sign of portal inflammation or fibrosis (Picture 2). During operation her liver was looking normal with adequate volume and remnant, Right lobe was taken out without middle hepatic vein. She tolerated procedure well. Her post-operative recovery was uneventful. Patient also recovered well.

Conclusion: There are not enough similar studies, this case report has shown that in selected cases, post bariatric surgery obese person can also be considered for liver donation safely, though larger studies needed to confirm it.
\end{abstract}

Keywords: Non-Alcoholic Fatty Liver Disease (NAFLD); Liver Transplantation; Chronic Liver Disease

\section{Introduction}

Liver transplantation is considered as a definitive treatment for cirrhotic patients. Scarcity of organs remains the most common problem in liver transplantation, which leads to increased death rate of patients in waiting list. Extended criteria were introduced to overcome this.

Steatotic liver grafts represent the most common type of "extended criteria" organs.

Hepatic steatosis is one of the most common causes of an increasing prevalence of non-alcoholic fatty liver disease (NAFLD)
[1]. NAFLD is one of the most common causes of chronic liver disease, found among $30 \%$ of individuals in Western countries and 70 - $80 \%$ of obese persons [2,3].

Weight reduction by bariatric surgery can prevent the progression of NAFLD to chronic liver disease $[4,5]$. Accepting post bariatric surgery obese liver donor was a big challenge to us, as there are not enough publications prior to this.

\section{Case Report}

Patient was 50 year old male, diagnosed having cryptogenic chronic liver disease two year ago. He started decompensating 
with ascites, coagulopathy, requiring multiple episodes of hospitalization due to intractable ascites, renal dysfunction and coagulopathy. His CTP score was C11 and MELD score was 33. After evaluation, he was advised liver transplant. Initially his 23 year old son came forward as a liver donor, but on evaluation he was found to be having fatty liver with raised liver enzymes. He was given dietary and exercise regimen but did not respond to it, repeat tests were also shown worsen results. As patient's condition was worsening, family was advised to look for another donor in family. His 50 year old wife was refused for donation, as she was having past history of bariatric surgery (Laparoscopic sleeve gastrectomy 6 year ago) and her recent BMI was also more then 35 , but as no other suitable donor was available in family and patient's condition was deteriorating; on her persistent request, her evaluation was started. Surprisingly her liver functions were absolutely normal, USG abdomen was normal. MR fat estimation showed 3 - 5\% fat fraction in her right and left lobe (Picture 1). Ct volumetric analysis showed adequate remnant and graft volume. Liver biopsy analysis showed 5 - 7\% fat fraction without any sign of portal inflammation or fibrosis (Picture 2 and 3). After her cardiopulmonary evaluation she was selected as prospective liver donor for her husband. After legal committee clearance liver transplant was done. She received preoperative prophylactic dose for venous thromboembolism. She was kept on DVT pump during and after surgery. During operation her liver was looking normal with adequate volume and remnant, liver edges were sharp. Right lobe was taken out without middle hepatic vein. Considering her safety, no segment 4 vein was ligated. She tolerated procedure well. Her post-operative recovery was very smooth. Her liver enzymes SGOT maximum 365 was on POD 1 and SGPT was 319 on Postoperative day 2 (Chart 1), maximum INR was 2.44 on post-operative day 2 (Chart 2). She was started on diet on postoperative day 2 . There was no bile leak. She was discharged on postoperative day 5 . Her recipient also showed smooth recovery. Except minor bile leak, there was no major post-operative complication. His immunosuppression was started as per routine protocol, induction with steroids and then combined with tacrolimus and mycophenolate mophetil. He was discharged on postoperative day 18.

\section{Discussion}

Donor safety should be priority while doing living donor liver transplant. As steatotic liver can lead to NAFLD, thorough evaluation of donor liver is must before liver transplant.

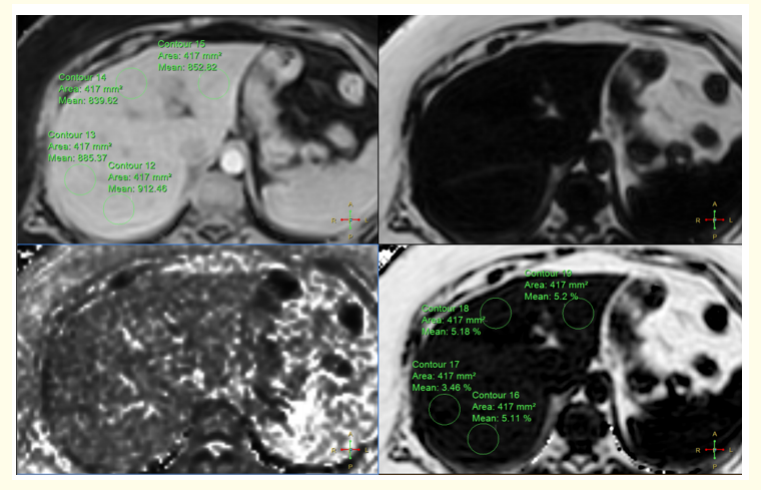

Picture 1: MR Fat fraction by two point Dixon method- Right lobe $7.35 \%$, left lobe $5.7 \%$.

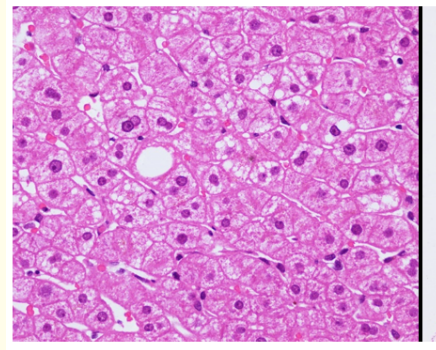

Right Lobe

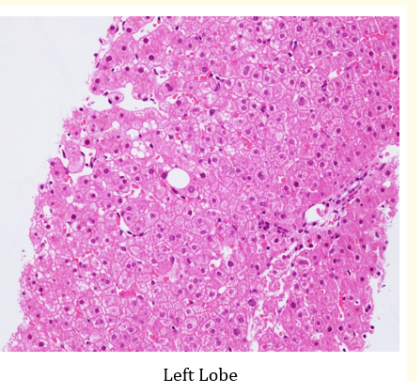

Left Lobe
Picture 2: Liver biopsy right lobe 5 - 8\%, Left lobe $3-5 \%$ fat fraction.

Steatosis in liver grafts increases complications after LT [6], such as prolonged ICU stay, hospital stay, the incidence of primary graft dysfunction or non-function and cost $[7,8]$.

Accurate liver fat estimation is a tough task for liver transplant team. Initially sonographic methods, provide qualitative information about liver steatosis, but are technically inadequate for quantitative assessment of liver fat. Non-contrast CT examination by liver attenuation index method (LAI) is used as a common tool with acceptable accuracy for assessing liver fat $[9,10]$.

MRI is now considered as a reliable option for non-invasive estimation of liver fat. Different MR techniques, like magnetic reso- 


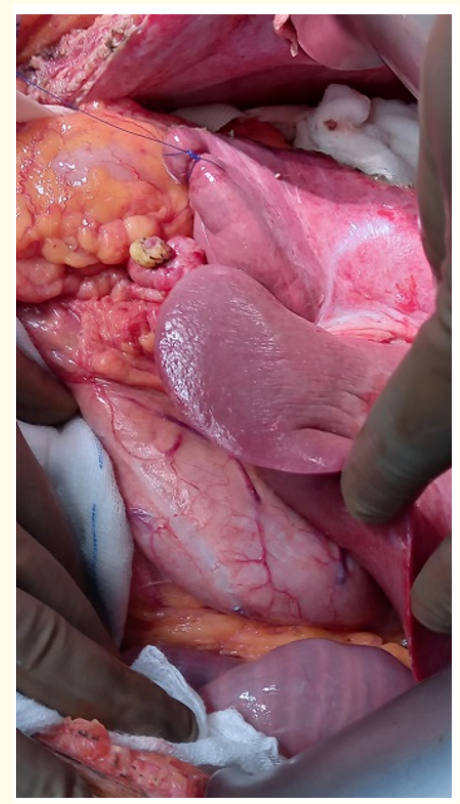

Picture 3: Intraoperative picture of donor hepatectomy showing sharp edges of liver with post sleeve gastrectomy stomach.

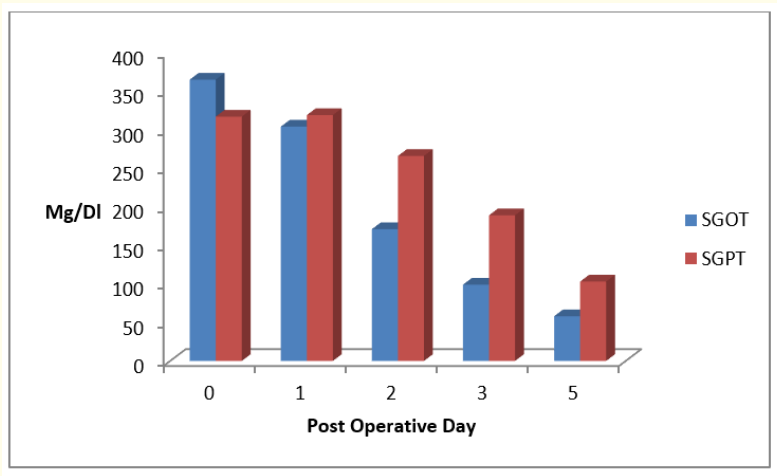

Chart 1: Post operative SGOT and SGPT level.

nance MR estimation of the liver using mDixon technique provide specific information about liver fat, correlated well with the histological grading. It does not involve ionizing radiation; technique is more accurate than CT [11].

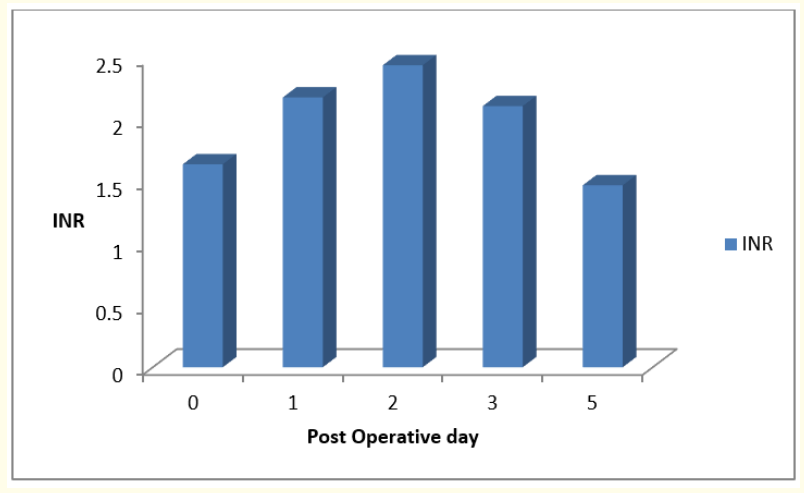

Chart 2: Post-operative INR.

The gold standard to assess hepatic steatosis is a histological analysis by a pathologist [12].

We quantified donor liver fat by doing CT LAI, MR fat estimation by two point dixon method. We routinely don't do liver biopsy in donor. As a protocol we do MR fat estimation in every case and as per our past experience, we observed a very good correlation between MR fat estimation and liver biopsy. To be on safer side we did liver biopsy from both lobes of liver to assess steatosis and inflammation.

Our donor regain approximately $20 \%$ weight in 6 year duration post bariatric surgery, but on liver biopsy there was only minimal steatosis and no inflammation. Previous studies have already shown that bariatric surgery is an efficient treatment of obesity and causes sustained weight loss with potential reductions in hepatic fat, inflammation and fibrosis [13,14].

This case report has shown that with good case selection; thorough investigations, preoperative and postoperative care, post bariatric surgery obese person can also be considered for liver donation safely, though larger studies needed to confirm it.

\section{Bibliography}

1. Nocito A., et al. "When is steatosis too much for transplantation?" Journal of Hepatology 45 (2006): 494-499.

2. Amarapurkar DN., et al. "How common is non-alcoholic fatty liver disease in the Asia-Pacific regionand are there local dif- 
ferences?" Journal of Gastroenterology and Hepatology 22 (2007): 788-793.

3. Chitturi S., et al. "Non-alcoholic fatty liver disease in the AsiaPacific region: definitions and overview of proposed guidelines". Journal of Gastroenterology and Hepatology 22 (2007): 778-787.

4. Luo RB., et al. "How bariatric surgery affects liver volume and fat density in NAFLD patients". Surgical Endoscopy (2017).

5. Moretto M., et al. "Effect of bariatric surgery on liver fibrosis". Obesity Surgery 22.7 (2012): 1044-1049.

6. McCormack L., et al. "Use of severely steatotic grafts in liver transplantation: a matched case-control study". Annals of Surgery 246 (2007): 940-946.

7. Seifalian AM., et al. "In vivo demonstration of impaired microcirculation in steatotic human liver grafts". Liver Transplant Transplant Surgery 4 (1998): 71-77.

8. Kaibori M., et al. "Liver regeneration in donors evaluated by Tc-99m-GSA scintigraphy after living donor liver transplantation". Digestive Diseases and Sciences 53 (2008): 850-855.

9. Angulo P., et al. "Independent predictors of liver fibrosis in patients with nonalcoholic steatohepatitis". Hepatology 30.6 (1999): 1356-1362.

10. Limanond P., et al. "Macrovesicular hepatic steatosis in living related liver donors: correlation between CT and histologic findings". Radiology 1 (2004): 276-280.

11. Venkatraman Bhat., et al. "Quantification of Liver Fat with mDIXON Magnetic Resonance Imaging, Comparison with the Computed Tomography and the Biopsy". Journal of Clinical and Diagnostic Research 11.7 (2017): TC06-TC10.

12. El-Badry AM., et al. "Assessment of hepatic steatosis by expert pathologists: the end of a gold standard". Annals of Surgery 250 (2009): 691-697.

13. Madsbad S., et al. "Mechanisms of changes in glucose metabolism and bodyweight after bariatric surgery". The Lancet Diabetes and Endocrinology 2 (2014): 152-164.
14. Lassailly G., et al. "Bariatric Surgery Reduces Features of Nonalcoholic Steatohepatitis in Morbidly Obese Patients". Gastroenterology 149 (2015): 379-388.

\section{Volume 4 Issue 6 June 2021}

(C) All rights are reserved by Manoj Gupta., et al. 